\title{
Grenzwertig: die Fortsetzung der EU-Erweiterung als Stabilitätsexport
}

\author{
Barbara Lippert*
}

\section{Osterweiterung als Erfolgsgeschichte}

Der substanzielle Beitrag der Europäischen Union zur Neuordnung Europas nach dem Ende des Ost-West-Konflikts findet allgemein große Anerkennung. Die Europäische Union gestaltete maßgeblich die neuen Beziehungen zwischen Westeuropa und dem neuen Mittelosteuropa (MOE). Infolge der teils gewaltsamen Auflösung von Föderationen und im Zuge der Neu- und Wiedererlangung von staatlicher Souveränität veränderte sich mit dem Jahr 1989 die politische Landkarte im Zentrum und Osten Europas grundlegend. Als eine Art Leuchtturm gab die Europäische Union im Moment der Auflösung der alten Fronten und beim Entstehen einer neuen Ordnung Orientierung für die außen- wie die innenpolitische Neuausrichtung der Staaten Mittel- und Osteuropas. Ihr Hauptinstrument war das Angebot der Aufnahme neuer Mitgliedstaaten.

Formelhaft wird die (Ost-)Erweiterung als erfolgreichstes und effektivstes Instrument der EU-Außenpolitik bezeichnet. ${ }^{1}$ Denn die Europäische Union dehnte auf diesem Weg die Werte und Standards, auf denen sie gründet, auf andere europäische Staaten aus. Diese Grundlagen reichen von der Demokratie und Rechtsstaatlichkeit über die Regeln der Wettbewerbspolitik bis hin zu den Bestimmungen für die Lebensmittelsicherheit. Durch die Aufnahme neuer Mitgliedstaaten gewinnt die Europäische Union an Größe und potenziell an äußerer Macht, weil diese Konvergenzprozesse zu ihren Bedingungen und nach ihren Maßstäben stattfinden. Dieses Wachstum verschafft der Europäischen Union international mehr Gewicht, vor allem aber mehr Reputation. Denn sie wird als Beispiel einer erfolgreichen Gemeinschaftsbildung von Staaten angesehen, obwohl diese untereinander große Unterschiede aufweisen hinsichtlich Bevölkerungsgröße, Wirtschaftskraft, Geografie, Kultur und Geschichte. Für Drittstaaten liegt die Attraktion der erweiterten Union also vielfach in ihrer Integrationsleistung nach innen.

Die Projektion von ,soft power' durch die Europäische Union ergänzte die NATO ${ }^{2}$ nach 1989 durch die Gewährung einer Sicherheits- und Beistandsgarantie für die MOE-Staaten. Europäische Union und NATO verfolgten mit ihren je eigenen Programmen und Zeitplänen das gemeinsame Ziel der Integration der Nachbarn in die euro-atlantischen Sicherheitsstrukturen. Auch im Feld der Sicherheit bedeutete die Erweiterung einen Gewinn für alte und neue Mitgliedstaaten.

Die wohl größte und entscheidende Wandlungskraft entfaltete die Europäische Union aber im Innern der Kandidatenländer: Brüssel bot mit dem Heranführungsprozess in Verbindung

\footnotetext{
* Dr. Barbara Lippert, Forschungsdirektorin, Stiftung Wissenschaft und Politik, Berlin.

Der Vortragsstil des Beitrags zum Workshop „Der Wert Europas“ am 16. Januar 2012 im Auswärtigen Amt, Berlin, wurde beibehalten.

1 Vgl. zum Zusammenhang von Erweiterungspolitik und Außenpolitik der Europäischen Union Barbara Lippert: EU-Erweiterung. Vorschläge für die außenpolitische Flankierung einer Beitrittspause, Stiftung Wissenschaft und Politik: SWP-Studie 7/2011.

2 North Atlantic Treaty Organization.
} 
mit einem glaubwürdigen Beitrittsversprechen allen MOE-Staaten ein umfassendes Transformationsprogramm an. Diese Angebote entwickelten dann Dynamik, wenn es Akteure in den Kandidatenländer verstanden, sie in ihre nationalen Reformprogramme zu integrieren und so anzuverwandeln, dass daraus eine Perspektive für die Zukunft des eigenen Landes und für seinen Platz in Europa und der Welt erwuchs. Reformorientierte Eliten ergriffen die EU-Option als Alternative zu rückwärtsgewandten Politikangeboten der kommunistischen und vorkommunistischen Zeit. Der Beitritt war für sie ein „Fluchtweg aus ihrer Vergangenheit und eine Sicherheitspolice für die Zukunft". ${ }^{3}$ Die innere wie äußere Dimension zusammen genommen, machten die EU-Erweiterung 2004/07 zum Kern der Neuordnung Europas nach dem Fall der Mauer.

\section{Europa nach 1989 ohne Erweiterung - volatiles Zwischeneuropa}

Hätten sich die demokratisch legitimierten Eliten in den MOE-Staaten in den Revolutionsjahren um 1989 nicht eindeutig für die ,Rückkehr nach Europa' mit der Europäischen Union als Referenzpunkt und Anker für Demokratie und Modernisierung in Staat und Gesellschaft entschieden und hätte die Europäische Union ihrerseits nicht relativ zügig (Gipfel in Kopenhagen 1993) die strategische Entscheidung für die Erweiterung um alle Staaten des PHARE-Raums ${ }^{4}$ getroffen, wären die letzten 20 Jahre in Europa turbulenter und konfliktreicher verlaufen. Die Schwäche der Sowjetunion beziehungsweise Russlands, die Wucht der Veränderungen in den vormals kommunistischen Staaten und die zügigen Erfolge der konstitutionell-institutionellen Systemtransformation in den meisten MOE-Staaten boten damals (einmalig) günstige Voraussetzungen dafür, dass die Konzepte, Politikinhalte und Instrumente der Europäischen Union greifen und die Europäische Union den Nachbarschaftsraum weitgehend politisch-institutionell strukturieren konnte. Gestützt auf diese günstige Konstellation konnte die Europäische Union eine Ordnungsvorstellung für den Kontinent im Maßstab des PHARE-Raums entwickeln, ohne selbst eine Transformation zu einem klassischen, staatsähnlichen Akteur in der Außen- und Sicherheitspolitik durchlaufen zu müssen. Wo im engeren Sinne hingegen sicherheitspolitische Kapazitäten gefragt waren, wie beim kriegerischen Auseinanderfallen Jugoslawiens, verpasste die Europäische Union die ,Stunde Europas', verstand dies aber zumindest als Weckruf, um Fähigkeiten und Konzepte für eine Europäische Sicherheits- und Verteidigungspolitik zu entwickeln.

Die Osterweiterung hat ein großes, vagabundierendes Zwischeneuropa verhindert und bis hin zur heutigen Enklave der Westbalkan-Staaten die Staaten auf den Pfad der Integration geführt. In gewissem Sinne war diese Rundum-Lösung durch Beitritt zu bestehenden Institutionen (Europarat, Europäische Union, NATO) für beide Seiten die einfachste. Alternativarrangements wären denkbar gewesen und wurden, wie François Mitterrands Vorschlag für eine gesamteuropäische Konföderation, kurzzeitig verfolgt. Man hätte zum Beispiel um den Kern der wirtschaftlich-politischen Assoziierung mit der Europäischen Union ein Netzwerk von weiteren sektoralen Abkommen knüpfen und andere Organisationen wie die Konferenz über Sicherheit und Zusammenarbeit in Europa (KSZE)/Organisation für Sicherheit und Zusammenarbeit in Europa (OSZE) stärker ins Spiel bringen oder neue Sicherheitsorganisationen schaffen und einen gesamteuropäischen Wirtschaftsraum zum Hauptanliegen ma-

3 Tony Judt: Geschichte Europas von 1945 bis zur Gegenwart, Bonn 2006, S. 851.

4 PHARE steht für „Poland and Hungary: Aid for Restructuring of the Economies“. Das Programm wurde 1989/90 aufgelegt und richtete sich an die zehn MOE-Staaten, die schließlich 2004 beziehungsweise 2007 beigetreten sind sowie das damalige Jugoslawien. 
chen können. Das hätte im Einzelfall gegebenenfalls gute Lösungen ermöglicht, aber insgesamt eine sehr viel höhere Volatilität und geringere Erwartungsverlässlichkeit zwischen den europäischen Staaten mit sich gebracht. Das, was jetzt als Integrationskonkurrenz zwischen Moskau und Brüssel um die östlichen Staaten der Europäischen Nachbarschaftspolitik (ENP) aufscheint, ${ }^{5}$ hätte - etwa mit Blick auf die Baltischen Staaten - zu einem Dauerbrennpunkt in einem neuen Ost-West-Konfliktraum werden können. Die Rolle der USA in Mittel- und Osteuropa wäre heute erheblich größer, der Einfluss der Europäischen Union jedoch geringer. In dem Szenario ,Europa nach 1989 ohne Erweiterung' hätten Volatilität und Spannungen auch in höherem Maße die innere Entwicklung in den Beitrittsstaaten gekennzeichnet. Mit dem Beitritt zur Europäischen Union und NATO haben die mittelosteuropäischen Nachbarn auf Dauer Entscheidungen zu allen Grundsatzfragen auf den Feldern Herrschaft, Wohlfahrt und Sicherheit getroffen. Diese Fundamentalentscheidung für die Europäische Union entlastete die Akteure innenpolitisch, sie förderte zumindest bis zum EUBeitritt die Kompromiss- und Konsensbildung, allerdings auch eine Elite, die sich zunächst auf Roadmaps und Programme externer Akteure stützte und vielfach nach dem Beitritt einen neuen Politikmodus finden und mit neuen innenpolitischen Konfliktlinien umgehen muss.

\section{Die Zukunft der Erweiterung - Europe whole and free?}

Kann oder soll die Europäische Union diese Erfolgsgeschichte durch die Aufnahme aller Staaten fortsetzen, die einen Antrag auf Mitgliedschaft stellen oder dies anstreben? Das für die Erweiterung ins Feld geführte Torso- beziehungsweise Einheits-Argument, nach dem Europa beispielsweise ohne die Ukraine nicht vollendet sei oder die Vision von einem ,Europe whole and free', wie sie amerikanische Präsidenten seit George Bush Senior propagieren, ist nicht evident. Denn was die Vollendung Europas wäre, hat die Europäische Gemeinschaft/Europäische Union nie prinzipiell und abschließend beantwortet, sondern immer bis auf Weiteres politisch untereinander vereinbart.

Die Erweiterung ist - auch in ihrem reaktiven Grundmuster - vor allem Ausdruck dessen, was die Europäische Union dem eigenen Selbstverständnis nach sein will und sein soll. Dieses Rollenkonzept der normativen Macht ließ und lässt eine bloß realistische außenpolitisch kalkulierte Erweiterungspolitik nicht zu. ${ }^{6}$ Da die Europäische Union sich aber selbst zum Preis macht, den sie an Kandidaten für deren erfolgreiche Transformation entrichtet, hat sie ein enormes Interesse an einer strikten Bindung der Aufnahme an umfassende Konditionen, wie sie der Europäische Rat auf dem Gipfel von Kopenhagen 1993 festgelegt und seitdem, etwa im erneuerten Konsens über die Erweiterung 2006, ausgebaut und verstärkt hat. ${ }^{7}$

Für die nächste Etappe der europäischen Ordnungsbildung ist nicht von einer linearen Fortschreibung der Erweiterung auszugehen, selbst wenn die Europäische Union nach Kroatien auch die übrigen Staaten des Westbalkans aufnehmen dürfte und damit ihre auf dem

5 Zum Begriff der Integrationskonkurrenz siehe Astrid Sahm: Nach der Wahl ist vor der Wahl. Belarus weiter auf Isolationskurs?, in: Osteuropa 1/2005, S. 77-90, hier S. 88.

6 Zum Konzept der normativen Macht vgl. Ian Manners: Normative Power Europe: A Contradiction in Terms?, in: Journal of Common Market Studies 2/2002, S. 235-258.

7 Rat der Europäischen Union: Tagung des Europäischen Rates vom 21./22. Juni 1993 in Kopenhagen. Schlussfolgerungen des Vorsitzes, Dok. 180/1/93; Rat der Europäischen Union: Tagung des Europäischen Rates vom 14./15. Dezember 2006 in Brüssel. Schlussfolgerungen des Vorsitzes, Dok. 16879/1/06. 
Gipfel von Thessaloniki 2003 eingegangene politische Selbstverpflichtung einlösen wird. ${ }^{8}$ Gegenüber ihrer östlichen Nachbarschaft versucht die Europäische Union, sich von dieser pfadabhängigen Entwicklung zu lösen. Sie verfolgt dort seit der Großerweiterung 2004/07 eine Doppelstrategie: Gestützt auf die Erfahrung der Erweiterungspolitik besteht diese erstens aus dem Export des eigenen Regelwerks (,external governance'), ohne dem Zielland eine explizite Beitrittsperspektive einzuräumen. Die Übernahme großer Teile des EUAcquis, besonders im Bereich Freihandel, Wettbewerb und flankierende Politiken, macht den Kern der heutigen ENP aus. Zweitens und parallel setzt die Europäische Union ihre klassischen außenpolitischen Instrumente zur Konfliktregulierung und zur Stabilisierung von Transformationsstaaten ein. Diese Komponente ist deutlich schwächer ausgebildet und sollte künftig größeres Gewicht erhalten. Denn während die Fähigkeiten der Union im Bereich der Gemeinsamen Außen- und Sicherheitspolitik (GASP) und der Gemeinsamen Sicherheits- und Verteidigungspolitik (GSVP) für die zurückliegende Osterweiterung nur in Maßen relevant waren (siehe allein die parallele NATO-Expansion), spielt die sicherheitspolitische Dimension auch wegen der Hegemonialansprüche Moskaus gegenüber der gemeinsamen Nachbarschaft heute eine sehr viel größere Rolle. Vor diesem Hintergrund hätte es gute Gründe dafür gegeben, die Hohe Vertreterin der Union für Außen- und Sicherheitspolitik (HV), Catherine Ashton, und nicht wie jetzt einen Kommissar mit der Nachbarschaftspolitik zu betrauen. In der Gemeinsamen Mitteilung von Catherine Ashton und Štefan Füle zur revidierten ENP finden sich auch kaum Anzeichen für ein stärkeres außenpolitisches Engagement. ${ }^{9}$

Zudem fehlt den Nachbarschaftsbeziehungen - auf Seiten der Europäischen Union wie der sechs europäischen ENP-Staaten und Russlands - die Fundierung durch eine gemeinsame substanzielle Vorstellung davon, wie die innere Ordnung der Staaten und die Beziehungen untereinander aussehen sollen. Im Hinblick auf eine normative Basis sind in jüngerer Zeit sogar eher Rückschritte in die Richtung autoritärer und repressiver Regime zu verzeichnen gewesen, so in Belarus, Aserbaidschan und der Ukraine. Im Falle von Georgien und Moldova sieht die innenpolitische Bilanz zwar positiver aus. Ihr stehen jedoch die Territorial- und Souveränitätskonflikte gegenüber, die weiterhin einen Hemmschuh für die innere Entwicklung bedeuten und eine sehr viel konstruktivere Kooperation zumindest zwischen der Europäischen Union und Russland erfordern würden.

Es fehlt weiterhin eine Architektur, die Sicherheit ungeteilt für alle europäischen Staaten garantiert. Die östliche Nachbarschaft der Europäischen Union bleibt ordnungspolitisch umstritten und geopolitisch labil.

Vor diesem Hintergrund ist der „Mehr für mehr“-Ansatz Brüssels in der ENP derzeit für die ENP-Staaten nicht besonders attraktiv. Das EU-Angebot der schrittweisen wirtschaftlichen Integration, der finanziellen wie immateriellen Unterstützung und der politischen Assoziierung wird von den meisten östlichen ENP-Staaten nicht mit besserer Regierungsführung, mehr Rechtsstaatlichkeit, Freiheit und Achtung der Menschenrechte beantwortet und damit mehr oder minder, verdient'. Positiv sind Anstrengungen der Europäischen Union zu werten, neben den politisch-administrativen Eliten zunehmend gesellschaftliche oder nicht-

8 Vgl. Barbara Lippert: EU-Erweiterung: Das Restprogramm, in: Annegret Bendiek/Barbara Lippert/Daniela Schwarzer (Hrsg.): Entwicklungsperspektiven der EU. Herausforderungen für die deutsche Europapolitik, Stiftung Wissenschaft und Politik: SWP-Studie 18/2011, S. 118-129.

9 Europäische Kommission/Hohe Vertreterin der Europäischen Union für Außen- und Sicherheitspolitik: Gemeinsame Mitteilung an das Europäische Parlament, den Rat, den Europäischen Wirtschafts- und Sozialausschuss und den Ausschuss der Regionen. Eine neue Antwort auf eine Nachbarschaft im Wandel, KOM (2011) 303. 
staatliche Akteure in die Kooperation, aber auch die öffentliche und transparente Bewertung der Entwicklung der Beziehungen einzubinden. Bislang hat sich die Europäische Union nicht zu einer konsequenteren Handhabung ihrer Prinzipien, also auch nicht zu einem ,Weniger für weniger', durchringen können. Eine solche leistungsabhängige Behandlung könnte beispielsweise innerhalb eines Liga-Systems praktiziert werden, das den Aufstieg wie den Abstieg kennt. Die Grundidee wäre, die ENP-Staaten des Ostens (wie des Südens) im Rahmen einer strikten Konditionalitätspolitik in unterschiedliche Ligen einzuordnen. Die Qualität der jeweiligen Beziehungen richtete sich nach den Leitlinien der Integration, Kooperation oder bloßer Kontakte, Letzteres definierte eine Art Mindestsockel der Beziehungen. Die Ligen würden durch diversifizierte Angebotskörbe und Implementierungsanforderungen ausgestaltet. ${ }^{10}$ Von der Zielsetzung der Europäischen Union, eine „,vertiefte Demokratie“"11 zu entwickeln, sind die Nachbarstaaten im Osten und Süden der Europäischen Union noch weit entfernt. Mehr noch als für den Westbalkan gilt, dass alle beteiligten Akteure in einem längeren Zeithorizont an der praktischen Kooperation und der Herausbildung einer gemeinsamen Wertebasis arbeiten müssen. Ihren Wert als Ordnungsfaktor wird die Europäische Union weiterhin vor allem in Europa unter Beweis stellen müssen.

\section{Deutschland in Europa}

Gerade Deutschland hat in puncto Freiheit und Sicherheit wie auch bei Handel und industrieller Arbeitsteilung von der Überwindung der europäischen Spaltung beträchtlich und in der ,EU-15‘ wohl am stärksten profitiert. Bei voller Berücksichtigung innenpolitischer Faktoren (siehe Arbeitnehmerfreizügigkeit) konnte Deutschland durch sein konsequentes Eintreten für die Erweiterung der Europäischen Union zudem sein politisches Kapital mehren. Dabei wurde eine Antagonisierung Russlands vermieden, was auch künftig im deutschen Interesse sein wird.

Ein Szenario ,Europa nach 1989 ohne Erweiterung ‘ hätte für Deutschland wohl weniger wirtschaftliche Nachteile zur Folge gehabt als vor allem dauernde politische Belastungen mit sich gebracht. Denn mit dem Fall der Mauer war die deutsche Frage in ganz Europa wieder da und damit das Misstrauen von alten EU-Partnern und östlichen Nachbarn gegenüber einer Zentralmacht Deutschland, die sich in MOE durch wirtschaftliche Durchdringung und politische Abhängigkeiten einen Hinterhof schaffen und gegebenenfalls diesen zusammen mit Russland dominieren würde. Die Grundfrage nach Hegemonie und Gleichgewicht in Europa sollte - gerade aus der deutschen Interessenlage heraus - erneut integrativ, durch den EU-Beitritt insbesondere der Visegrád-Staaten gelöst werden. ${ }^{12}$ Die Bilateralismen nicht als Sonderbeziehungen Berlins, sondern im EU-Rahmen zu entwickeln, wirkte den Sorgen

10 Vgl. die Ausformulierung dieser Idee in Kai-Olaf Lang/Barbara Lippert: Zur Neuausrichtung der ENP: Ein Liga-Modell nachbarschaftlicher Kooperation, in: Annegret Bendiek/Barbara Lippert/Daniela Schwarzer (Hrsg.): Entwicklungsperspektiven der EU. Herausforderungen für die deutsche Europapolitik, Stiftung Wissenschaft und Politik: SWP-Studie 18/2011, S. 102-117; Kai-Olaf Lang/Barbara Lippert: The EU and Its Neighbours, SWP Comments 2/2012; vgl. dagegen mit Blick auf die Süd-ENP-Staaten Muriel Asseburg: Proteste, Aufstände und Regimewandel in der arabischen Welt. Akteure, Herausforderungen, Implikationen und Handlungsoptionen, Stiftung Wissenschaft und Politik: SWP-Studie 27/2011.

11 Europäische Kommission/Hohe Vertreterin: Eine neue Antwort, 2011, S. 2.

12 Vgl. Werner Link: Auf dem Weg zu einem neuen Europa. Herausforderungen und Antworten, Baden-Baden 2006. 
aller Nachbarn entgegen und hat Deutschlands außen- und europapolitische Handlungsspielräume in keiner Weise geschmälert. ${ }^{13}$

Allerdings war dafür ein europapolitischer Preis zu zahlen. Mit der Erweiterung war ein Verzicht auf die Option beziehungsweise die Drohkulisse ,Kerneuropa“ verbunden; vor allem war aber - wie im Zeitablauf deutlich wurde - zumindest ein Abbremsen der Vertiefungsdynamik zu einer „,immer engeren Union der Völker Europas“ ${ }^{\text {“14 }}$ zu verzeichnen. Die Integrationsverlangsamung und stärkere Binnendifferenzierung in der erweiterten und heterogeneren Union traf seit Mitte der 1990er Jahre zusammen mit wachsenden Legitimitätsproblemen des EU-Systems insgesamt. Zwar liegt ein Zurückdrehen der Osterweiterung 2004/07, wie es Frankreich unter Nicolas Sarkozy bei der laufenden Reform der Governance in der Eurozone in Betracht zog (und was Polen unter anderem fürchtet), nicht im deutschen Interesse. Wohl aber eine Europäische Union, die auf längere Sicht nur die Staaten des Westbalkans aufnehmen und sich darüber hinaus als Ordnungsfaktor ohne Rückgriff auf die Erweiterung so gut es geht behaupten wird. In der Perspektive der ,EU-28 plus` sollte Deutschland der Verdichtung der Integration einschließlich einer effektiven GASP und der Verbesserung von Demokratie und Legitimität der Union selbst Priorität beimessen.

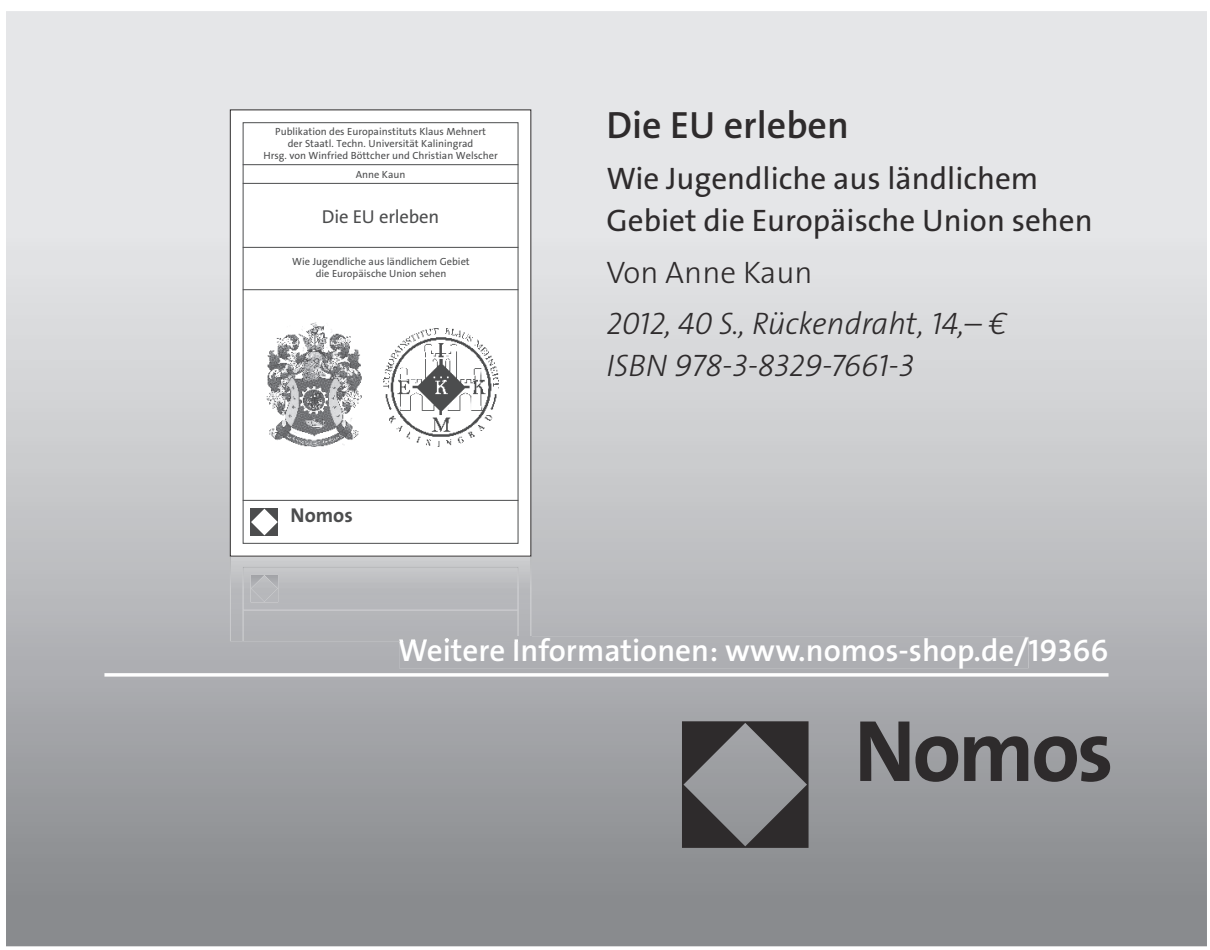

13 Vgl. auch in diesem Sinne das Papier von Wolfgang Schäuble/ Karl Lamers: Überlegungen zur europäischen Politik, 1.9.1994, abrufbar unter: http://www.cducsu.de/upload/schaeublelamers94.PDF (letzter Zugriff: 19.3.2012).

14 Präambel des Vertrags über die Europäische Union. 